\title{
Biofilm Models to Study the Etiology and Pathogenesis of Oral Diseases
}

Thurnheer, Thomas ; Paqué, Pune Nina

DOI: https://doi.org/10.1159/000510197

Posted at the Zurich Open Repository and Archive, University of Zurich

ZORA URL: https://doi.org/10.5167/uzh-195984

Book Section

Accepted Version

Originally published at:

Thurnheer, Thomas; Paqué, Pune Nina (2021). Biofilm Models to Study the Etiology and Pathogenesis of Oral Diseases. In: Eick, Sigrun. Oral Biofilms. Basel: Karger, 30-37.

DOI: https://doi.org/10.1159/000510197 
Monographs in Oral Sciences

\begin{tabular}{|l|l|}
\hline Manuscript: & MOS-2020-5-4 \\
\hline Title: & Biofilm models to study etiology and pathogenesis of oral diseases \\
\hline Authors(s): & $\begin{array}{l}\text { Thomas Thurnheer (Corresponding author), Pune Nina Paqué (Co- } \\
\text { author) }\end{array}$ \\
\hline Keywords: & $\begin{array}{l}\text { fluorescence in situ hybridization (FISH), biofilm models, confocal } \\
\text { laser scanning microscopy (CLSM), subgingival, supragingival }\end{array}$ \\
\hline Type: & Review Article \\
\hline
\end{tabular}




\section{Biofilm models to study etiology and pathogenesis of oral diseases}

Thomas Thurnheer *, Pune Paqué

Clinic of Conservative and Preventive Dentistry, Center of Dental Medicine,

University of Zurich, Zurich, Switzerland

Running Title: Biofilm models in oral diseases

\section{*Correspondence:}

Dr. Thomas Thurnheer, PhD

Division of Oral Microbiology and Immunology, Clinic of Conservative and Preventive Dentistry, Center of Dental Medicine, University of Zurich

Plattenstrasse 11, 8032 Zurich, Switzerland

E-mail: thomas.thurnheer@zzm.uzh.ch

Tel: +41446343376 


\section{Abstract}

More than 700 microbial species inhabit the complex environment of the oral cavity. Since years microorganisms have been studied in pure cultures, a highly artificial situation because microorganisms in natural habitats grow as complex ecologies, termed as biofilms. These resemble multi-cellular organisms and are characterized by their overall metabolic activity upon multiple cellular interactions. Microorganisms in biofilms express different genes than their planktonic counterparts resulting in higher resistance to antimicrobials, different nutritional requirements, or creation of a low redox potential allowing the growth of strictly anaerobic bacteria in the presence of oxygen. Multiple in vitro biofilm models have been described in literature so far. The main emphasis here will be on multi-species biofilm batch culture models developed in Zurich. The standard six-species supragingival biofilm model has been used to study basic aspects of oral biofilms such as structure, social behavior and spatial distribution of microorganisms, or diffusion properties. Numerous parameters related to inhibition of dental plaque were tested illustrating the high reliability of the model to predict the in vivo efficiency of antimicrobials. Modifications and advancements led to a ten-species subgingival model often combined with human gingival epithelial cells, as an integral part of the oral innate immune system, eliciting various cell responses ranging from cytokine production to apoptosis. In summary, biofilm models enable to address a multitude of questions that cannot be studied with planktonic monocultures. The Zurich in vitro biofilm models are reproducible and reliable and may be used for studies of basic, but also for application-oriented questions that could not be addressed using culture techniques. Oral biofilm research will certainly lead to a more realistic assessment of the role of microorganisms in the oral cavity in health and disease. In this respect, substantial progress has been made but still, there is more to explore. 
Keywords: biofilm models, supragingival, subgingival, confocal laser scanning microscopy (CLSM), fluorescence in situ hybridization (FISH) 


\section{Introduction}

The oral cavity is a complex environment and home to more than 700 microbial species (1). For many years, the oral ecosystem was studied using planktonically growing organisms in order to investigate and understand all the different components of this ecosystem. Although the fact that microorganisms are able to grow attached to solid surfaces was reported already in 1936 by Zobell (2), it took more than 40 years until it was recognized that in nature most bacteria grow in biofilms attached to a surface rather than growing planktonically $(3,4)$.

According to Costerton and modified later by IUPAC, a biofilm is defined as a structured community of microbial cells embedded in a self-produced hydrated matrix of extracellular polymeric substances (EPS), which is adherent to an inert or living surface $(4,5)$. When grown in a biofilm, microbial cells differ physiologically from planktonic cells of the same organism, which are swimming or floating single cells in a liquid medium. A cell switching to the biofilm mode of growth undergoes a phenotypic shift in behavior with many genes being differentially regulated (6). Biofilms may be formed in response to factors such as recognition of attachment sites on a surface, nutritional signals or protection from harmful conditions (7-9). Living in a biofilm represents a universal survival strategy of microorganisms on our planet, allowing them to colonize new ecological niches and survive in hostile environments, thereby adopting biofilm structure in response to environmental conditions $(10,11)$. The dense and perplex/complex structure of a biofilm not only hampers diffusion of molecules but also forms a barrier against the host defense mechanisms such as antibodies, lysozyme, or against other antimicrobial agents. 
For many years, the microbial species of the oral ecosystem were studied individually. However, for the reasons described above and in order to understand how microorganisms form biofilms as well as their diverse functions in this environment, it was essential to develop novel experimental models of oral biofilms. Since the late nineties of the last century, multiple in vitro biofilm models have emerged, each of them especially adapted to observe biofilm formation of specific bacteria within specific environments or applied to answer questions of clinical relevance, most notably biofilm permeability and chemical control of dental plaque. Some aspects that are of interest are spatial arrangement and associative behavior of various bacterial species in biofilms; mass transport in biofilms; the biofilm model as a reliable tool to predict the in vivo efficacy of antimicrobials, and de- and remineralization of enamel exposed to biofilms in vitro. In order to establish biofilm models a clear understanding of processes involved in biofilm formation and its pathogenicity is essential.

\section{In vitro modelling of oral biofilms}

To date, multiple in vitro biofilm models have emerged and described in the last two decades. Oral biofilm models can be divided into three groups: constant depth film fermenter, flow cell chamber systems, and closed batch culture models. The constant depth film fermenter is a dynamic biofilm model that allows the control of environmental factors such as the substratum, the nutrient source, the gas flow and especially biofilm thickness $(12,13)$. The flow cell chamber system consists of a glass slide coated with saliva that is placed in a chamber and is crossed by a continuous flow of medium (14). So far, this model has been used in order to test the effect of osteopontin, a glycosylated and highly phosphorylated whey protein in multispecies biofilms (15). Furthermore, a flow chamber model was used to examine 
the effect of antibiotics on established biofilms and allows for the observation of biofilm formation under flow and shear force conditions (16). With batch biofilm models, a biofilm is formed either on a plate wall, on the surface of discs, coupons or pegs or on human/bovine enamel within the well. A closed system is used so that the environment inside the well changes during the test as nutrients are consumed and metabolic products accumulate unless the growth media are replaced (17). While constant-depth film fermenter biofilm models and flow cell chamber systems work under flow conditions and closely mimic in vivo situation or show real-time biofilm formation, respectively, and thus have contributed to our understanding of microbial adhesion and biofilm formation, their use has certain drawbacks. For instance, they can be cumbersome to construct and/or difficult to maintain over long periods of time. Since clearance of pulsed substances is a function of flow rate and volume, chemostats operating with low flow rates and relatively large volumes can have quite long mean residence times, rendering them impractical for studies of selected compounds with short-term exposure, as is common in oral hygiene procedures. Moreover, systems with working volumes of more than a few milliliters preclude the use of media constituted from natural substrates such as saliva.

Depending on the aim of biofilm analyses, the design of the model can vary considerably with regards to the substrate, medium, biofilm harvesting and subsequent analysis. In some studies, glass or polystyrene surfaces are used as substrates for biofilm formation, whereas dentin, enamel or artificial hydroxyapatite discs are also used in other reports. For biofilm growth, the medium used has to fulfill the complex nutritional requirements. Saliva or combinations of saliva with selective media can be applied as well. To identify biofilm microorganisms either cultivationdependent or cultivation-independent approaches are applied. 


\section{Biofilm models to study etiology and pathogenesis of oral diseases}

Artificial subgingival biofilms can be generated in the microbiology laboratory in order to study their behavior in vitro and deduce conclusions for their behavior in vivo. These biofilm models can be clinically very useful in understanding the role of different biofilm bacteria in the pathogenesis of diverse diseases and in testing the antimicrobial efficiency of different antimicrobial agents prior to clinical application. According to earlier studies (18), there are several requirements that should be met by a good model system. Most importantly, results generated by the model system should be reproducible.

Due to the enormous microbial complexity of periodontal disease, there are several obstacles to overcome in order to successfully build a sufficient model system. The overwhelming amount of different bacterial species, as well as the morphological diversity, is a limiting factor that restricts the accurate matching of the in vivo situation with a biofilm model system.

\section{The supragingival biofilm model}

The Zurich biofilm model has been established more than a decade ago and was designed as a fully defined, in vitro batch model system used as a supragingival model consisting of six oral microorganisms characteristic for supragingival plaque (19-22). This model was later extended and thereby modified to a subgingival model (23-28) (see below). In Fig. 1 confocal laser scanning microscopy (CLSM) images of three versions of the Zurich biofilm model are depicted. The first version of the supragingival model contains five different species (Actinomyces naes/undii, Veillonella dispar, Fusobacterium nucleatum, Streptococcus sobrinus, and Streptococcus oralis). The biofilms were developed on hydroxyapatite discs coated with pasteurized human saliva for 64 hours in anaerobic conditions before collection 
(19). This model was subsequently improved by adding a yeast, Candida albicans, in order to improve the correspondence between the model's response to antimicrobial agents and the effects of these agents in vivo $(22,25)$. The procedures for biofilm production have been described in detail before $(22,25,29)$. In brief, the standard supragingival biofilms were grown anaerobically in 24-well polystyrene cell culture plates on hydroxyapatite discs that had been preconditioned for pellicle formation in whole unstimulated pooled saliva (in the following termed saliva) for $4 \mathrm{~h}$. To initiate a biofilm, experiment discs were covered for the first $16 \mathrm{~h}$ with $1.6 \mathrm{ml}$ of growth medium containing $70 \%$ saliva, $30 \%$ modified fluid universal medium (mFUM) supplemented with Sørensen's buffer (final pH 7.2) and $200 \mu$ of a cell suspension prepared from equal volumes and densities of each strain. The medium was changed after $16 \mathrm{~h}$ and $40 \mathrm{~h}$. For the first $16 \mathrm{~h}$, the medium contained $0.3 \%$ glucose. After 16 $\mathrm{h}$ the medium was replenished with one containing $0.15 \%$ glucose and $0.15 \%$ sucrose, instead of $0.3 \%$ glucose. In order to remove non-adherent microorganisms, biofilms were dipped three times in saline after $16 \mathrm{~h}, 20 \mathrm{~h}$ and $24 \mathrm{~h}$ as well as after $40 \mathrm{~h}, 44 \mathrm{~h}$ and $48 \mathrm{~h}$. After $64 \mathrm{~h}$ of incubation, the biofilms were dip-washed again and either harvested for culture analyses by vigorous vortexing in $1 \mathrm{ml}$ of $0.9 \% \mathrm{NaCl}$ or proceeded to staining and CLSM.

Figure $1 \mathrm{~A}$ shows a CLSM image of an in vitro supragingival biofilm. This biofilm is relatively thin compared to the biofilms in Figs $1 \mathrm{~B}$ and $1 \mathrm{C}$, respectively, and the cells seem loosely dispersed. The red-stained $F$. nucleatum can be observed throughout the biofilm biomass, implementing its role as a bridging organism between early and late colonizers (30).

The supragingival model has been used extensively to test the antimicrobial effectiveness of various components like plant extracts, polyphenolic compounds, and mouthwashes (22). The model was also used to examine the effect of fluoride in 
$\mathrm{NaF}$ formulation, on the microbiota and demineralization of enamel discs in vitro (31). Moreover, the supragingival model was used to investigate the three-dimensional architecture of the biofilm allowing us to gain an insight into the structural features of all species during biofilm development, and into the associative behavior of the strains within the biofilm (21). Biofilms were stained with DNA stains to visualize total bacteria or selectively stained by fluorescence in situ hybridization (FISH) using specific rRNA-targeted probes $(20,32)$. When such biofilms were additionally stained with the exopolysaccharide stain Calcofluor (33), it became evident that multispecies biofilms formed in the presence of oral streptococci and sucrose consist of microbial microcolonies embedded in a compact polysaccharide hydrogel (20,32, 33). Diffusion experiments with such double-stained biofilms revealed that dextrans larger than $10 \mathrm{kD}$ cannot pass the extracellular polysaccharide moiety directly and must find their way on winding pathways through microcolonies. On the other hand, smaller molecules seemed to find their way through the biofilm unhamperedly (33). These findings may explain the lower cariogenic potential of starch in comparison to lowmolecular-weight saccharides.

\section{The supragingival 'feeding' biofilm model}

In order to mimic more accurately the fast and feast periods experienced by natural dental plaque, the supragingival 'feeding' model termed as 'feeding' model was established (34). Therefore, the standard experimental protocol described above was modified as follows: (1) the proportion of saliva and mFUM was reversed to $30 \%$ saliva and $70 \%$ mFUM and (2) the exposure to this altered medium was time-limited. Meaning, after inoculation the discs remained for only 45 min in the feeding solution containing $0.3 \%$ glucose. Thereafter, they were subjected to three consecutive 1 -min washes in $2 \mathrm{ml} 0.9 \% \mathrm{NaCl}$ to remove growth medium and free-floating cells but not 
bacteria adhering firmly to hydroxyapatite discs. The biofilms were then further incubated in new wells containing $1.6 \mathrm{ml}$ of saliva and no mFUM. Only after $16 \mathrm{~h}, 20$ h, $24 \mathrm{~h}, 40 \mathrm{~h}, 44 \mathrm{~h}$ and $48 \mathrm{~h}$, the biofilms were pulse-fed by transferring the discs for 45 min into $30 \%$ saliva/ $70 \%$ mFUM with $0.15 \%$ glucose and $0.15 \%$ sucrose. Thereafter, they were washed as described above and reincubated in saliva. Fresh saliva was provided after $16 \mathrm{~h}$ and $40 \mathrm{~h}$, respectively. After $64 \mathrm{~h}$, biofilms were washed and processed for further analyses.

These 'feeding' biofilms are more dense than supragingival biofilms generated by the batch biofilm model (see Fig. 1B) and adhere very strongly to the substrate. This model is therefore suitable for studies, investigating mechanical or hydrodynamic effects on biofilms. In this context,the 'feeding' biofilm model was used e.g. to investigate the biofilm removal capacity of ultrasonic scaler tips under standardized conditions $(29,35)$.

The subgingival biofilm model

In order to grow subgingival in vitro biofilms, the protocol for standard supragingival biofilms described above was modified as follows: (1) ten bacterial species were used instead of six, namely Actinomyces oris (OMZ 745), Campylobacter rectus (OMZ 388), Fusobacterium nucleatum ssp. nucleatum (OMZ 598), Porphyromonas gingivalis ATCC $33277^{\top}$ (OMZ 925), Prevotella intermedia ATCC $25611^{\top}(\mathrm{OMZ} 278)$, Streptococcus anginosus ATCC 9895 (OMZ 871), Streptococcus oralis SK 248 (OMZ 607), Tannerella forsythia (OMZ 1047), Treponema denticola ATCC $35405^{\top}(\mathrm{OMZ}$ 661), and Veillonella dispar ATCC $17748^{\top}$ (OMZ 493), (2) the growth medium contained $60 \%$ saliva, $10 \%$ fetal bovine serum, and $30 \%$ FUM. To generate subgingival biofilms, the same procedure as described for the standard supragingival biofilm model was applied. 
The ten bacterial species used in this model were selected according to published observations concerning biofilm formation and periodontal disease. The main goal was to incorporate the main disease-associated, 'red-complex' species (36). To facilitate their incorporation, the additional bacterial species were selected with the goal to provide a suitable matrix in terms of attachment receptors (37) and redox potential, while further nutritional conditions were optimized (25). The biofilms produced by the improved model system remarkably resembled their in vivo pendants in both structure and quantitative distribution of the species (23-25). The subgingival model system proved to produce stable and reproducible biofilms, alike both supragingival biofilm models. Additionally, the subgingival biofilms in proximity to cultured human epithelial cells induced cellular apoptosis (26), and a number of histopathological $(28,38)$ and protein changes known to be associated with periodontal diseases (39). The above described approach allowed for a direct link of primary human gingival epithelial cells, as an integral part of the oral innate immune system, to an in vitro subgingival biofilm, and thereby elicit various cell responses ranging from cytokine production to apoptosis.

In Figure 1C, a CLSM image of the subgingival biofilm model is shown. It is evident that the subgingival biofilm model results in much thicker and more dense biofilms than the supragingival biofilm models. Again, F. nucleatum (stained red) is spread throughout the biofilm biomass. Microcolonies of $P$. gingivalis (stained blue) can also be observed.

The subgingival biofilm model has been used in an in vitro study to investigate the colonization of human gingival multi-layered epithelium by multi-species subgingival biofilms, and evaluated the relative effects of the "red complex" species (P. gingivalis, $T$. forsythia and $T$. denticola) (28). In another in vitro study, the subgingival model has been slightly modified to develop an in vitro "submucosal" 
biofilm model for peri-implantitis by incorporation of staphylococci into titanium-grown biofilms (40).

\section{Conclusive remarks}

While various studies have described biofilm formation in static systems, bacteria in the oral cavity are subject to constantly changing environmental conditions (e.g., salivary flow). Static biofilm models are not able to simulate these conditions, and specific research questions require dynamic models. The use of the described biofilm

models allows to address a multitude of questions that cannot be studied with planktonic monocultures. The Zurich in vitro biofilm models are reproducible and reliable. They may be used for the study of basic, but also for application-oriented questions that could not be addressed using culture techniques.

Our data indicate that, compared to responses triggered by planktonic individual species, the bacteria organized in an in vitro subgingival biofilm express even more damaging virulence factors neutralizing the proinflammatory defense of host cells. As neither the culture of host defense cells, nor the assembly of artificial biofilms is restricted to oral tissues and bacteria, the same strategy of challenging cultured host cells with in vitro propagated bacterial biofilms may be of general interest and could be applied to study other elusive chronic inflammatory diseases.

\section{Acknowledgments}

We would like to thank Manuela Flury for the outstanding technical assistance during the experiments. We also thank the Center of Microscopy and Image Analysis (ZMB) of the University of Zurich for the supply of confocal laser scanning microscope (CLSM). 


\section{Conflict of Interest Statement}

The authors have no conflicts of interest to declare.

\section{Funding Sources}

The study was supported by Institutional funds of the University of Zurich.

\section{Author contributions}

TT wrote the manuscript, PP critically reviewed the manuscript. All authors read and approved the final manuscript.

\section{References}

1. Aas J, Paster B, Stokes L, Olsen I, Dewhirst F. Defining the normal bacterial flora of the oral cavity. J Clin Microbiol. 2005;43:5721-32.

2. Zobell C, DQ A. Observations on the multiplication of bacteria in different volumes of stored sea water and the influence of oxygen tension and solid surfaces. Biol Bull. 1936;71:324.

3. Costerton J, Geesey G, Cheng K. How bacteria stick. Sci Am. 1978;238:86-95.

4. Costerton JW, Lewandowski Z, Caldwell DE, Korber DR, Lappin-Scott HM. Microbial biofilms. Annu Rev Microbiol. 1995;49:711-45.

5. Vert M, Doi Y, Hellwich K-H, Hess M, Hodge P, Kubisa P, et al. Terminology for biorelated polymers and applications. Pure Appl Chem. 2012;84:377-410.

6. Khemiri A, Jouenne T, Cosette P. Proteomics dedicated to biofilmology: What have we learned from a decade of research. Med Microbiol Immunol. 2015.

7. Jefferson KK. What drives bacteria to produce a biofilm? FEMS Microbiol Lett. 2004;236(2):163-73.

8. Karatan E, Watnick P. Signals, regulatory networks, and materials that build and break bacterial biofilms. Microbiol Mol Biol Rev. 2009;73(2):310-47.

9. Stoodley P, Dodds I, Boyle J, Lappin-Scott H. Influence of hydrodynamics and nutrients on biofilm structure. J Appl Microbiol. 1998;85 Suppl 1:19S-28S.

10. Bowden GH, Hamilton IR. Survival of oral bacteria. Crit Rev Oral Biol Med. 1998;9(1):54-85.

11. Hall-Stoodley L, Costerton J, Stoodley P. Bacterial biofilms: from the natural environment to infectious diseases. Nat Rev Microbiol. 2004;2:95-108. 
12. Wilson M. Use of constant depth film fermentor in studies of biofilms of oral bacteria. In: Doyle RJ, editor. Methods in Enzymology 310. Methods in Enzymology 310. San Diego: Academic Press; 1999. p. 264-79.

13. Pratten J. Growing oral biofilms in a constant depth film fermentor (CDFF). Curr Protoc Microbiol. 2007; Chapter 1:Unit 1B.5.

14. Periasamy S, Kolenbrander P. Central role of the early colonizer Veillonella sp. in establishing multispecies biofilm communities with initial, middle, and late colonizers of enamel. J Bacteriol. 2010;192(12):2965-72.

15. Schlafer S, Raarup MK, Wejse PL, Nyvad B, Stadler BM, Sutherland DS, et al. Osteopontin reduces biofilm formation in a multi-species model of dental biofilm. PLoS One. 2012;7(8):e41534.

16. Oettinger-Barak O, Dashper S, Catmull D, Adams G, Sela M, Machtei E, et al. Antibiotic susceptibility of Aggregatibacter actinomycetemcomitans JP2 in a biofilm. J Oral Microbiol. 2013;5.

17. Coenye $\mathrm{T}$, Nelis $\mathrm{H}$. In vitro and in vivo model systems to study microbial biofilm formation. J Microbiol Methods. 2010;83(2):89-105.

18. Ammann T. Advancement and structural analysis of a subgingival biofilm model system [PhD]: PhD thesis, University of Zurich; 2013.

19. Guggenheim B, Giertsen E, Schüpbach P, Shapiro S. Validation of an in vitro biofilm model of supragingival plaque. J Dent Res. 2001;80(1):363-70.

20. Guggenheim B, Guggenheim M, Gmür R, Giertsen E, Thurnheer T. Application of the Zürich biofilm model to problems of cariology. Caries Res. 2004;38(3):212-22.

21. Guggenheim M, Shapiro S, Gmür R, Guggenheim B. Spatial arrangements and associative behavior of species in an in vitro oral biofilm model. Appl Environ Microbiol. 2001;67(3):1343-50.

22. Shapiro S, Giertsen E, Guggenheim B. An in vitro oral biofilm model for comparing the efficacy of antimicrobial mouthrinses. Caries Res. 2002;36:93-100.

23. Ammann T, W., Belibasakis G, N., Thurnheer T. Impact of early colonizers on in vitro subgingival biofilm formation. PLoS ONE. 2013;8 12:e83090.

24. Ammann T, Bostanci N, Belibasakis G, Thurnheer T. Validation of a quantitative real-time PCR assay and comparison with fluorescence microscopy and selective agar plate counting for species-specific quantification of an in vitro subgingival biofilm model. J Periodontal Res. 2013;48:517-26.

25. Ammann T, Gmur R, Thurnheer T. Advancement of the 10-species subgingival Zurich Biofilm model by examining different nutritional conditions and defining the structure of the in vitro biofilms. BMC Microbiol. 2012;12(1):227.

26. Guggenheim B, Gmur R, Galicia J, Stathopoulou P, Benakanakere M, Meier A, et al. In vitro modeling of host-parasite interactions: the 'subgingival' biofilm challenge of primary human epithelial cells. BMC Microbiol. 2009;9:280.

27. Thurnheer T, Bostanci N, Belibasakis G. Microbial dynamics during conversion from supragingival to subgingival biofilms in an in vitro model. Mol Oral Microbiol. 2016;31 (2):125-35. 
28. Thurnheer T, Belibasakis G, Bostanci N. Colonisation of gingival epithelia by subgingival biofilms in vitro: Role of "red complex" bacteria. Arch Oral Biol. 2014;59(9):977-86.

29. Thurnheer T, Rohrer E, Belibasakis G, Attin T, Schmidlin P. Static biofilm removal around ultrasonic tips in vitro. Clin Oral Investig. 2014;18(7):1779-84.

30. Kolenbrander PE, Andersen RN, Blehert DS, Egland PG, Foster JS, Palmer RJ, Jr. Communication among oral bacteria. Microbiol Mol Biol Rev. 2002;66(3):486-505.

31. Thurnheer T, Belibasakis $\mathrm{G}$. Effect of sodium fluoride on oral biofilm microbiota and enamel demineralization. Arch Oral Biol. 2018;89:77-83.

32. Thurnheer T, Gmür R, Guggenheim B. Multiplex FISH analysis of a six-species bacterial biofilm. J Microbiol Meth. 2004;56(1):37-47.

33. Thurnheer T, Gmür R, Shapiro S, Guggenheim B. Mass transport of macromolecules within an in vitro model of supragingival plaque. Appl Environ Microbiol. 2003;69:1702-9.

34. Thurnheer T, van der Ploeg J, Giertsen E, Guggenheim B. Effects of Streptococcus mutans gtfC deficiency on mixed oral biofilms in vitro. Caries Res. 2006;40:163-71.

35. Gartenmann S, Thurnheer T, Attin T, Schmidlin P. Influence of ultrasonic tip distance and orientation on biofilm removal. Clin Oral Investig. 2016;21:1029-36.

36. Socransky S, Haffajee A, Cugini M, Smith C, Kent RJ. Microbial complexes in subgingival plaque. J Clin Periodontol. 1998;25(2):134-44.

37. Kolenbrander PE, London J. Adhere today, here tomorrow: oral bacterial adherence. J Bacteriol. 1993;175(11):3247-52.

38. Belibasakis GN, Kast JI, Thurnheer T, Akdis CA, Bostanci N. The expression of gingival epithelial junctions in response to subgingival biofilms. Virulence. 2015;6(7):704-9.

39. Bostanci N, Bao K, Wahlander A, Grossmann J, Thurnheer T, Belibasakis G. Secretome of gingival epithelium in response to subgingival biofilms. Mol Oral Microbiol. 2015;30(4):323-35.

40. Thurnheer T, Belibasakis G. Incorporation of staphylococci into titanium-grown biofilms: an in vitro "submucosal" biofilm model for peri-implantitis. Clin Oral Implants Res. 2016;27(7):890-5. 


\section{Figure legend}

Figure 1. Confocal laser scanning microscopy images (CLSM) of supragingival (A), feeding (B), and subgingival (C) in vitro biofilms. Due to fluorescence in situ hybridization (FISH) staining, Fusobacterium nucleatum appears red (A-C), streptococci $(A, B)$ and Porphyromonas gingivalis $(C)$, respectively, blue, whereas bacteria appear green due to DNA staining with Sytox/YoPro 1. The biofilm base in the cross-section is directed towards the top view. Scale bars: $10 \mu \mathrm{m}(\mathrm{A}, \mathrm{B})$ and 20 $\mu \mathrm{m}(\mathrm{C})$, respectively. 


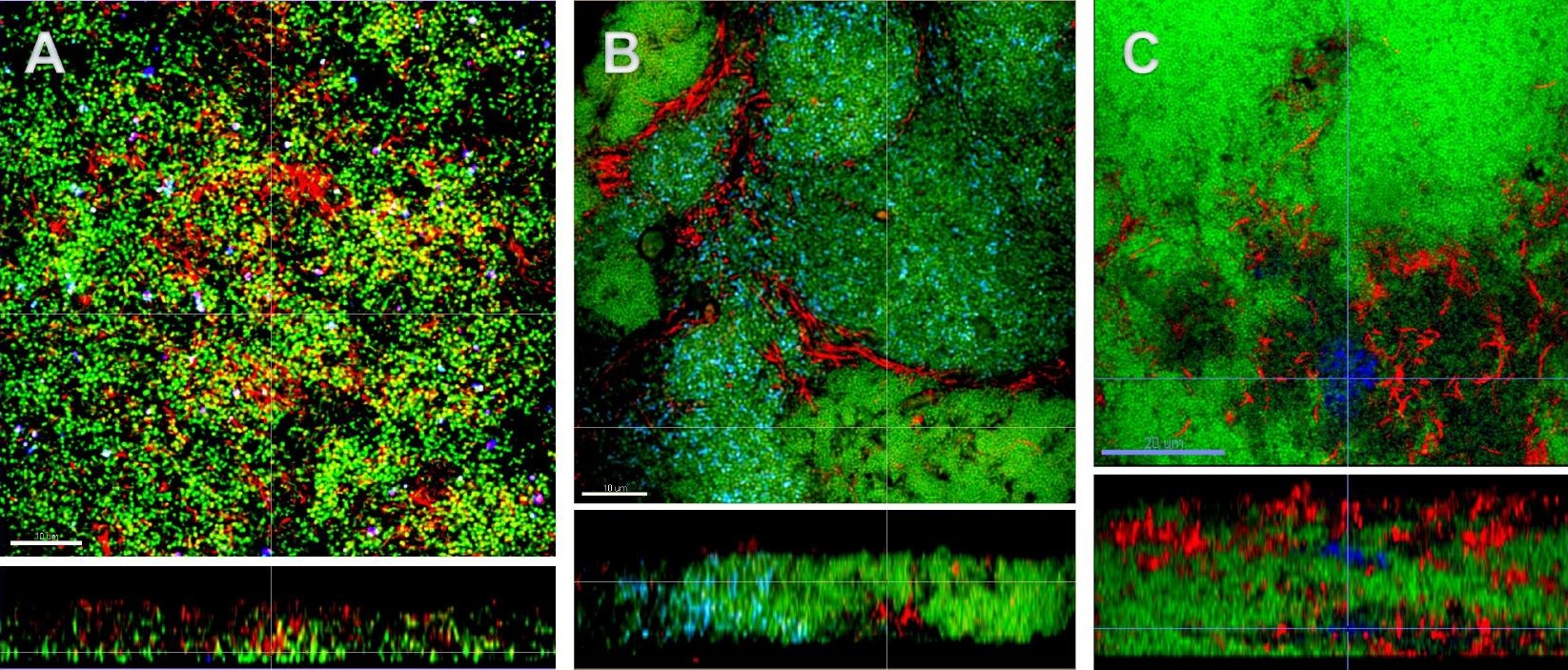




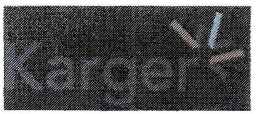

\section{Monographs in Oral Science}

\section{Submission Statement}

Manuscript Title:

Every author that has contributed to the submitted manuscript must sign this Submission Statement with his/her original signature.

By signing the Submission Statement, the author confirms that he / she has read and agreed to the following terms and conditions of this submission statement, including that

- the submission is original and has not been previously published

- all permissions have been obtained

- the manuscript includes all the relevant statements and acknowledgements

- the copyright is transferred to S. Karger AG on acceptance

\begin{tabular}{l|l|l|l|l} 
Printed Name: & E-Mail: & Signature: & Place and Date: \\
\hline Thomas Thurnheer & thomas.tnumherezzz. uzh.con & Zurich, 29 May 2020 \\
\hline Pune Paqué & punenina. paque@zzm. uzh.ch & & & \\
\hline & & & & \\
\hline & & & & \\
\hline & & & & \\
\hline
\end{tabular}

Please print and sign the form, and upload it during submission or fax or e-mail it to:

5. Karger AG - Medical and Scientific Publishers

Editorial Office Monographs in Oral Science

Allschwilerstrasse 10

$\mathrm{CH}-4009$ Basel (Switzerland)

Fax: +41613061434

E-Mail: a.lorenzekarger.com 